\title{
Topology Optimization of Frame Bracing System for Natural Frequency
}

\author{
Kemin Zhou* and Chahua Chen
}

College of Civil Engineering, Huaqiao University, Fujian, 361021, China

\begin{abstract}
A method to optimize the topology of frame bracing system is presented. Firstly frame is filled by truss-like continuum uniformly. The truss-like continuum simulates the distributive bracing system. The frame combined with trusslike continuum is analyzed by finite element method. The densities and orientations of bracing system at nodes are taken as design variables. The distribution field of bracing system is optimized by the method of moving asymptotes (MMA) and the method of steepest descent. The frame bracing system is established according to the optimal distribution field of bracing system. The natural frequency of optimal braced frame increases to more than two times while material increases only 5\%. For no intermediate densities being suppressed, there is no numerical instability, such as checkerboard patterns and one-node connected hinges. The expression of natural frequency and its sensitivities of truss-like continuum are derived.
\end{abstract}

Keywords: Bracing system, frame, natural frequency, structural optimization, topology optimization, truss-like continuum.

\section{INTRODUCTION}

Frame is used widely in structural engineering. Its later stiffness of frame is more important design factor than its strength. Especially when frame is acted by wind and earthquake, increasing the later stiffness and natural frequency of frame become the most important design object [1].

To improve structural performance, an effective mean is to optimize the sectional sizes of members and the structural shapes (the positions of nodes, for example). These optimization techniques are termed as size optimization and shape optimization, respectively. By these techniques structural performance is improved on the condition that the structural topology (i.e. how many members in structures and where these members are placed) is prescribed. These techniques are mature relatively and are applied widely in engineering already. Furthermore if structural topology can be changed, structural performance may be improved more greatly. However how to design structural topology is a difficult problem.

For a frame, bracing system has significant effect on its later stiffness [2-4]. For a complex frame, however, it is difficult to optimize the topology of bracing system. Generally X brace systems are adopted widely. However where and how many bracing members are placed is determined intuitively frequently. Fortunately the development of theory and method of topology optimization provide a powerful tool to overcome this difficulty.

Structural topology optimization was presented one hundred year ago [5]. The various numerical methods,

\footnotetext{
*Address correspondence to this author at the College of Civil Engineering, Huaqiao University, Fujian 361021, CHINA; Tel: +86-18965171319;

; E-mails: zhoukm@hqu.edu.cn and zhoukemin@tsinghua.org.cn
}

including homogenization method [6, 7], evolutionary structural optimization method $[8,9]$ and level set method [10], etc, have developed rapidly in recent decades. It is effective to design the reinforcement material by topology optimization method [11, 12]. In these optimization methods, isotropic continua are taken as ground structures. Structural topology is optimized by forming holes in uniform isotropic continua [13]. Furthermore, Mijar and Qing used evolutionary structural optimization method to optimize the bracing system of frame to minimize structural compliance $[2,3]$. Lots of elements are deleted in optimization procedure. The bracing system is expressed by a series of conjoint uniform isotropic elements remaining in structure. For this reason, to obtain detail bracing system, more elements are used. Therefore more numerical calculation work is needed. Furthermore, the numerical instabilities exist generally [14].

Michell's work has revealed the fact that topological optimal structures are truss-like continua generally [5]. Truss-like material model can express the optimal truss-like continuum accurately. By truss-like material model, the optimization problems for stress, compliance and natural frequency were studied $[15,16]$. In this paper, the optimization method is applied to optimize the frame bracing system.

\section{TRUSS-LIKE MATERIAL MODEL}

\subsection{Elasticity Matrix}

To optimize the topology of frame bracing system, the frame is filled by truss-like continuum fully, which stands for the distribution field of dense bracing system. The orientations and densities of members vary over whole design domain. To simplify this problem, it is assumed that there are two familiar members along with orthotropic 
orientations at any point. The densities of two familiars members are denoted as $t_{1}, t_{2}$, respectively. The relation between stress and strain is expressed as $\sigma_{b}=E t_{b} \varepsilon_{b}$ ( $b=1,2$ ), where $E$ is Young's modulus. Therefore the elasticity matrix can be written as,

$$
\boldsymbol{D}\left(t_{1}, t_{2}, 0\right)=E \cdot \operatorname{diag}\left[\begin{array}{lll}
t_{1} & t_{2} & \left(t_{1}+t_{2}\right) / 4
\end{array}\right]
$$

Here the shear stiffness $E\left(t_{1}+t_{2}\right) / 4$ is assumed so as to describe the isotropic material in the case of $t_{1}=t_{2}$. If the angles from global coordinates axis $x$ to members are denoted as $\alpha$ and $\alpha+\pi / 2$, with the aid of coordinates transform matrix

$\boldsymbol{T}(\alpha)=\left[\begin{array}{ccc}c^{2} & s^{2} & c s \\ s^{2} & c^{2} & -c s \\ -2 c s & 2 c s & c^{2}-s^{2}\end{array}\right], \begin{aligned} & c=\cos \alpha \\ & s=\sin \alpha\end{aligned}$

the elasticity matrix can be written as,

$\boldsymbol{D}\left(t_{1}, t_{2}, \alpha\right)=\boldsymbol{T}^{\mathrm{T}}(\alpha) \boldsymbol{D}\left(t_{1}, t_{2}, 0\right) \boldsymbol{T}(\alpha)=E \sum_{b=1}^{2} t_{b} \sum_{r=1}^{3} s_{b r} g_{r}(\alpha) \boldsymbol{A}_{r}$

where $s_{b r}$ are the constant $s_{11}=s_{12}=s_{13}=s_{23}=1, s_{21}=s_{22}=-1, g_{r}$ is the components of functional matrix $g$,

$\boldsymbol{g}(\alpha)=\left[\begin{array}{lll}\cos 2 \alpha & \sin 2 \alpha & 1\end{array}\right]$

$\boldsymbol{A}_{r}$ is constant matrix,

$\boldsymbol{A}_{1}=\frac{1}{2} \operatorname{diag}\left[\begin{array}{lll}1 & -1 & 0\end{array}\right]$, $\boldsymbol{A}_{2}=\frac{1}{4}\left[\begin{array}{ccc}0 & 0 & 1 \\ 0 & 0 & 1 \\ 1 & 1 & 0\end{array}\right]$

$\boldsymbol{A}_{3}=\frac{1}{2} \operatorname{diag}\left[\begin{array}{lll}1 & 1 & \frac{1}{2}\end{array}\right]$

Frame compounded with truss-like continuum is analyzed by finite element method. The densities $t_{b j}$ and orientations $\alpha_{j}$ of members in truss-like continuum at nodes are taken as design variables. According to (3), the elasticity matrix at node $j$ is expressed as a function of design variables $t_{b j}$ and $\alpha_{j}$,

$\boldsymbol{D}\left(t_{1 j}, t_{2 j}, \alpha_{j}\right)=E \sum_{b=1}^{2} t_{b j} \sum_{r=1}^{3} s_{b r} g_{r}\left(\alpha_{j}\right) \boldsymbol{A}_{r}$

The elasticity matrix at any point in an element is calculated by the interpolation of elasticity matrixes (6) at nodes belonging to the element,
$\boldsymbol{D}_{e}(\xi, \eta)=\sum_{j \in S_{e}} N_{j}(\xi, \eta) \boldsymbol{D}\left(t_{1 j}, t_{2 j}, \alpha_{j}\right)$

where $N_{j}(\xi, \eta)$ is shape function, $\xi, \eta$ is local coordinates, $S_{e}$ is the set of nodes belonging to the element $e$. Introducing (6) into (7) leads the elasticity matrix in the element $e$,

$\boldsymbol{D}_{e}=E \sum_{j \in S_{e}} N_{j} \sum_{b=1}^{2} t_{b j} \sum_{r=1}^{3} s_{b r} g_{r}\left(\alpha_{j}\right) \boldsymbol{A}_{r}$

\subsection{Stiffness Matrix and Its Sensitivities}

Introducing (8) into the definition of elementary stiffness matrix $\boldsymbol{k}_{e}=\int_{V_{e}} \boldsymbol{B}^{\mathrm{T}} \boldsymbol{D}_{e} \boldsymbol{B} \mathrm{d} V$ leads,

$\boldsymbol{k}_{e}=E \sum_{j \in S_{e}} \sum_{b=1}^{2} t_{b j} \sum_{r=1}^{3} s_{b r} g_{r}\left(\alpha_{j}\right) \int_{V_{e}} N_{j} \boldsymbol{B}^{\mathrm{T}} \boldsymbol{A}_{r} \boldsymbol{B} \mathrm{d} V=\sum_{j \in S_{e}} \sum_{b=1}^{2} t_{b j} \sum_{r=1}^{3} s_{b r} g_{r}\left(\alpha_{j}\right) \boldsymbol{H}_{e j r}$

where $\boldsymbol{B}$ is geometry matrix, $V_{e}$ is the whole element, $\boldsymbol{H}_{e j r}=E \int_{V_{e}} N_{j} \boldsymbol{B}^{\mathrm{T}} \boldsymbol{A}_{r} \boldsymbol{B} \mathrm{d} V$ is a constant matrix, which is independent of design variables. It can be calculated and stored before finite element analysis if regular rectangular element is used. Summation of (9) over all elements gives the global stiffness matrix,

$$
\boldsymbol{K}=\sum_{e} \boldsymbol{k}_{e}=\sum_{e} \sum_{j \in S_{e}} \sum_{b=1}^{2} t_{b j} \sum_{r=1}^{3} s_{b r} g_{r}\left(\alpha_{j}\right) \boldsymbol{H}_{e j r}=\sum_{j} \sum_{e \in S_{j}} \sum_{b=1}^{2} t_{b j} \sum_{r=1}^{3} s_{b r} g_{r}\left(\alpha_{j}\right) \boldsymbol{H}_{e j r}
$$

where $S_{j}$ is the set of elements around node $j$. The second equation in (10) comes from the exchange of the first summation order. Differentiating (10) with respect to design variables gives the sensitivities of stiffness matrix,

$\frac{\partial \boldsymbol{K}}{\partial t_{b j}}=\sum_{e \in S_{j}} \sum_{r=1}^{3} s_{b r} g_{r}\left(\alpha_{j}\right) \boldsymbol{H}_{e j r}$

and,

$\frac{\partial \boldsymbol{K}}{\partial \alpha_{j}}=\sum_{e \in S_{j}} \sum_{b=1}^{2} t_{b j} \sum_{r=1}^{2} s_{b r} g_{r}^{\prime}\left(\alpha_{j}\right) \boldsymbol{H}_{e j r}$

where $g_{r}^{\prime}\left(\alpha_{j}\right)$ is the derivation of the second equation in (4)

$\boldsymbol{g}^{\prime}\left(\alpha_{j}\right)=2\left[\begin{array}{lll}-\sin 2 \alpha_{j} & \cos 2 \alpha_{j} & 0\end{array}\right]$

\section{NATURAL FREQUENCY AND ITS SENSITIVITIES}

The frequency equation can be expressed as a general eigenvalue problem,

$\boldsymbol{M v}=\boldsymbol{K} \boldsymbol{v} / \omega^{2}$, or $\left(\boldsymbol{K} / \omega^{2}-\boldsymbol{M}\right) \boldsymbol{v}=0$

where $\omega$ and $v$ are the natural frequency and mode of vibration, respectively. The natural frequency can be calculated by Rayleigh quotient, 
$\frac{1}{\omega^{2}}=\frac{\boldsymbol{v}^{\mathrm{T}} \boldsymbol{M} \boldsymbol{v}}{\boldsymbol{v}^{\mathrm{T}} \boldsymbol{K} \boldsymbol{v}}$, or $\frac{1}{\omega^{2}} \boldsymbol{v}^{\mathrm{T}} \boldsymbol{K} \boldsymbol{v}=\boldsymbol{v}^{\mathrm{T}} \boldsymbol{M} \boldsymbol{v}$

For the amplitude of the mode of vibration being pointless, the mode $\boldsymbol{v}$ can be set to $\boldsymbol{v}^{\mathrm{T}} \boldsymbol{K} \boldsymbol{v}=1$. Therefore (15) can be written as

$1 / \omega^{2}=\boldsymbol{v}^{\mathrm{T}} \boldsymbol{M v}$

To maximize the natural frequency of vibration, deriving (16) with respect to design variables $t_{b j}$ and $\alpha_{j}$ leads,

$\frac{\partial}{\partial t_{b j}} \frac{1}{\omega^{2}}=-\frac{1}{\omega^{2}} \boldsymbol{v}^{\mathrm{T}} \frac{\partial \boldsymbol{K}}{\partial t_{b j}} \boldsymbol{v}, \frac{\partial}{\partial \alpha_{j}} \frac{1}{\omega^{2}}=-\frac{1}{\omega^{2}} \boldsymbol{v}^{\mathrm{T}} \frac{\partial \boldsymbol{K}}{\partial \alpha_{j}} \boldsymbol{v}$

Introducing (11) and (12) into(17), the sensitivities of natural frequency are obtained.

\section{OPTIMIZATION PROBLEM}

With the aid of shape function, the densities of members at any point in an element are interpolated by the densities of members at nodes belonging to the element $t_{b}(\xi, \eta)=\sum_{j \in S_{e}} N_{j}(\xi, \eta) t_{b j}$. The material volume is calculated by the integration of densities of members over the element $\sum_{b=1}^{2} \sum_{j \in S_{e}} \int_{V_{e}} N_{j} t_{b j} \mathrm{~d} V$. The total material volume of structure $V$ is calculated by the summation of material over all elements

$V=\sum_{e} \sum_{b=1}^{2} \sum_{j \in S_{e}} \int_{V_{e}} N_{j} t_{b j} \mathrm{~d} V$

The objective function is to maximize the natural

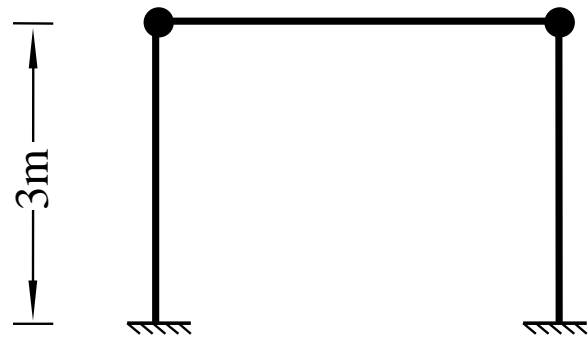

(a)

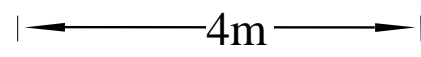

Fig. (1). 1-bay, 1-story frame (a) Structure (b) Finite element model. frequency with prescribed material volume,

$$
\begin{array}{lll}
\text { find } & t_{b j}, \alpha_{j} & b=1,2 ; \\
\min & 1 / \omega^{2} & \quad \\
\text { s.t. } & V \leq \bar{V}, t_{b j} \geq t &
\end{array}
$$

where $n$ is the number of nodes, $\bar{V}$ is the prescribed material volume, $\underline{t}$ is the low limit of densities, which is set to a little value to avoid the stiffness matrix becoming singular.

The densities of members at all nodes are optimized by the method of moving asymptotes (MMA) [17]. The orientations of members at all nodes are optimized by the steepest descent method. Densities and orientations are optimized alternatively until the relative change of design variables is small enough ( $1 \%$ in this paper).

\section{NUMERICAL EXAMPLES}

Four-node rectangular elements are used to mesh the truss-like continuum. Its volume is kept constant in optimization process. Young's modulus is 200GPa. Material density is $7850 \mathrm{~kg} / \mathrm{m}^{3}$.

The first example is a 1-bay, 1-story frame shown in Fig. (1a). Two concentrated mass of $10^{5} \mathrm{~kg}$ locate the two corners of frame. Column and beam use I-sections (Chinese National Standards GB/T 11263-2005) as shown in Table 1. Frame is divided by 20 beam elements; and truss-like continuum is meshed by rectangular elements of $8 \times 6$, as shown in Fig. (1b). The densities and orientations of members at nodes are optimized to minimize the structural frequency. Frequency increases stably in iteration process as shown in Fig. (3). Its natural frequency is increased considerably as shown in Table $\mathbf{2}$. The optimal truss-like continuum distribution is shown in Fig. (2a). The orientations and lengths of lines in Fig. (2a) stand for the orientations and densities of members in truss-like

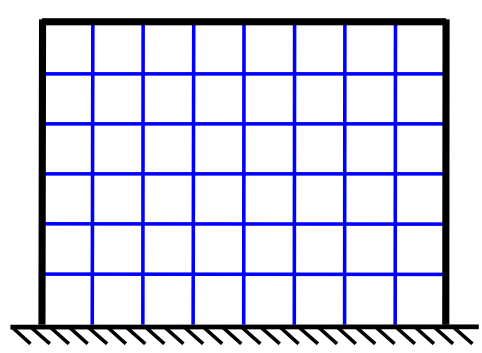

(b)

\begin{tabular}{|c|c|c|c|}
\hline & Section & A & I \\
\hline Column & HM194×150×6×9 & 39.76 & 2740 \\
\hline Beam & $\mathrm{HN} 300 \times 150 \times 6.5 \times 9$ & 47.53 & 7350 \\
\hline
\end{tabular}

Table 1. Sectional properties of example 1. 
continuum at nodes, respectively. To make the figure legible, some too long lines are cut. According to the optimal bracing system distribution field in Fig. (2a), optimal bracing system is suggested in Fig. (2b). In order to be fabricated easily, the structure in Fig. (2b) is simplified to the structure in Fig. (2c) as final optimal braced frame.

The second example is a 1-bay, 3-story frame shown in Fig. (4a). There are 5 groups of members with I-sections (Chinese National Standards GB/T 11263-2005) are used as shown in Table 3 . Frame is divided by 60 beam elements; and truss-like continuum is meshed by rectangular elements of $8 \times 18$, as shown in Fig. (4b). Frequency increases process in iteration is shown in Fig. (2). The optimal bracing system is illustrated in Fig. (5a). According to the optimal bracing system distribution field in Fig. (5a), optimal bracing system is formed in Fig. (5b). In order to be fabricated easily, the structure in Fig. (5b) is simplified to the structure in Fig. (5c) as final optimal braced frame.

The third example is a 2-bay, 6-story frame shown in

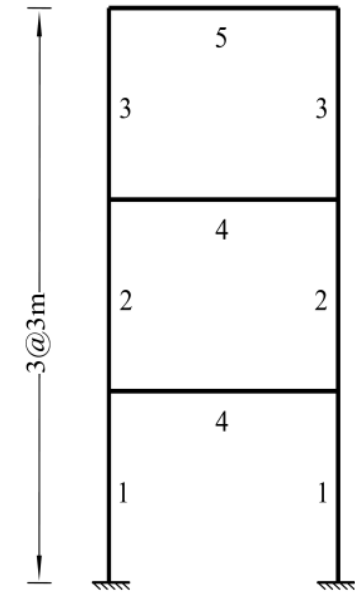

(a)

Fig. (4). 1-bay, 3-story frame (a) Structure and the number of member groups. (b) Calculation model.

Table 2. Sectional properties of example 2.

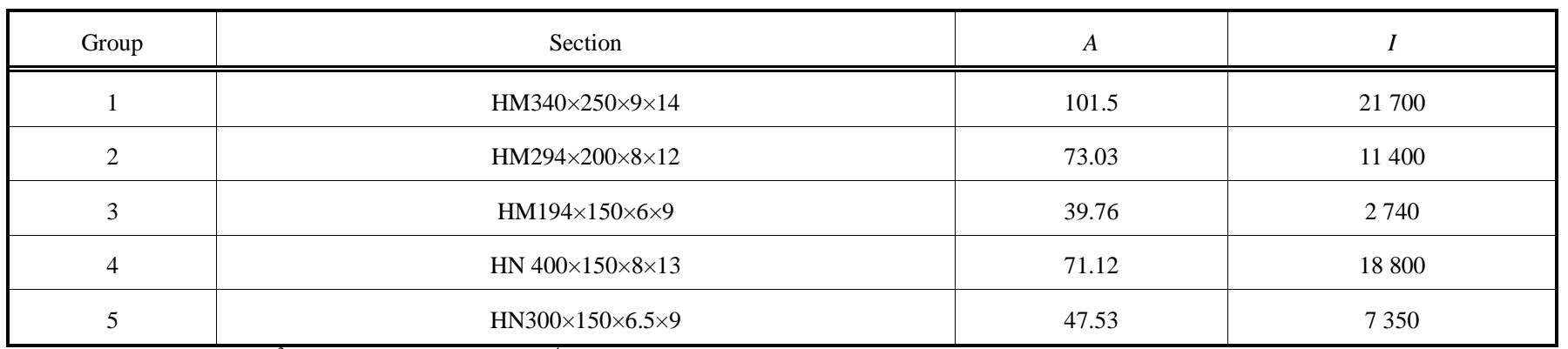

A: Cross-sectional area $\left(\mathrm{cm}^{2}\right) ;$ I: Moment of inertial $\left(\mathrm{cm}^{4}\right)$.

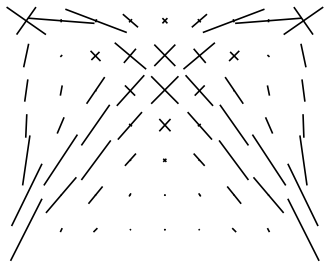

(a)

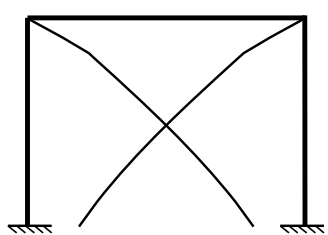

(b)

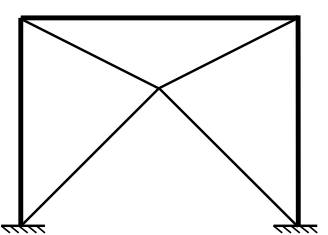

(c)

Fig. (2). Optimal results (a) Optimal truss-like continuum (b) Optimal bracing systems (c) Modified bracing systems.

Example 1

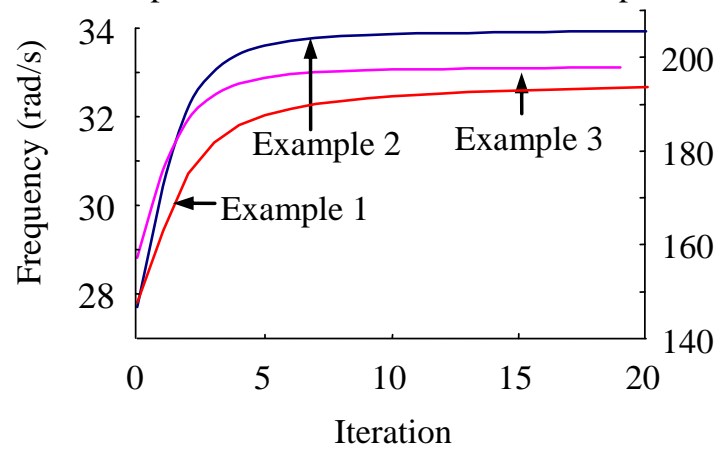

Fig. (3). Iteration history. 
Table 3. Sectional properties of example 3.

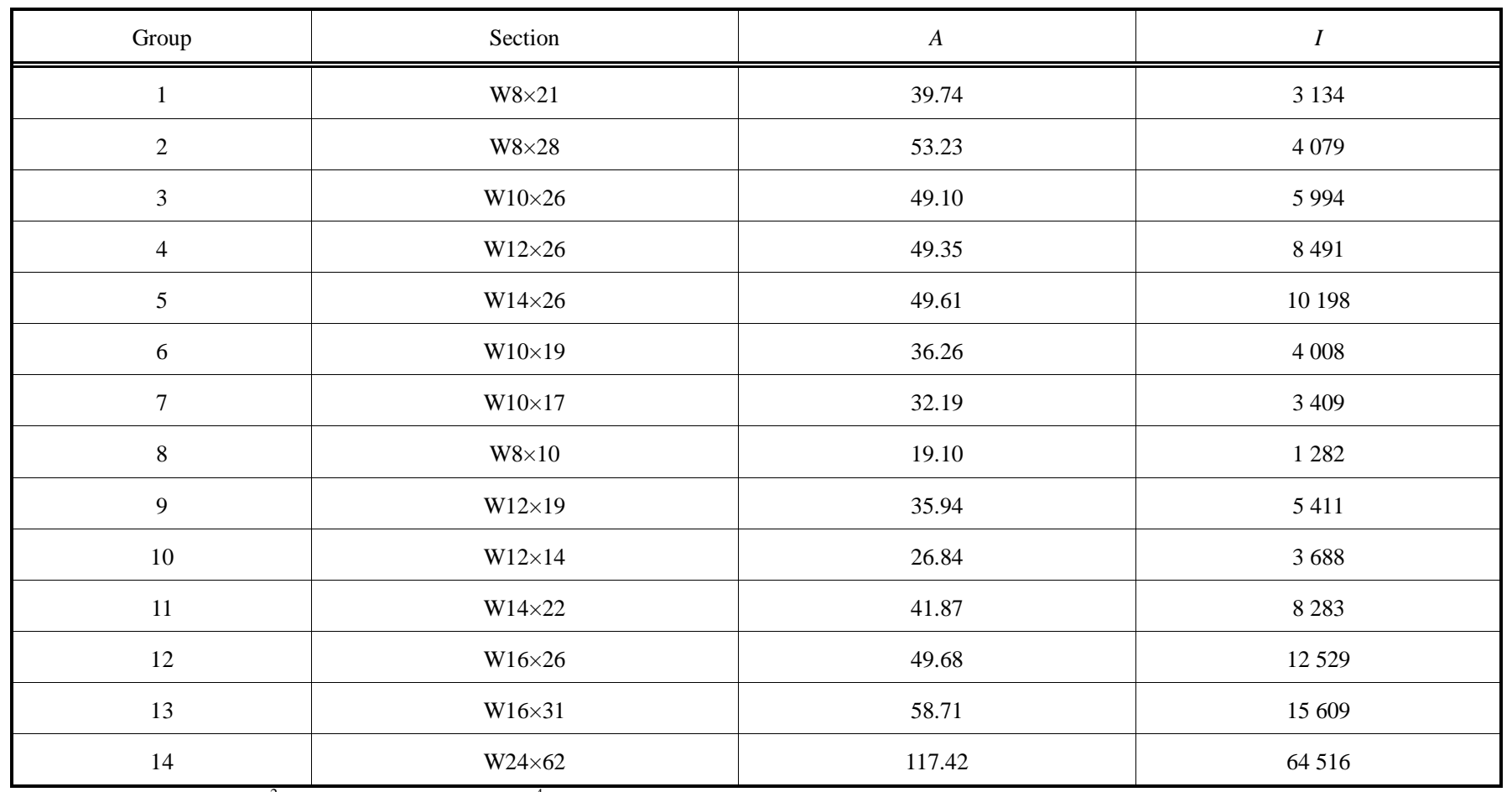

A: Cross-sectional area $\left(\mathrm{cm}^{2}\right) ;$ : Moment of inertial $\left(\mathrm{cm}^{4}\right)$

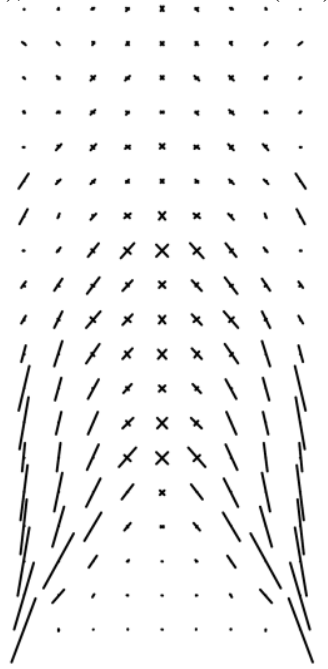

(a)

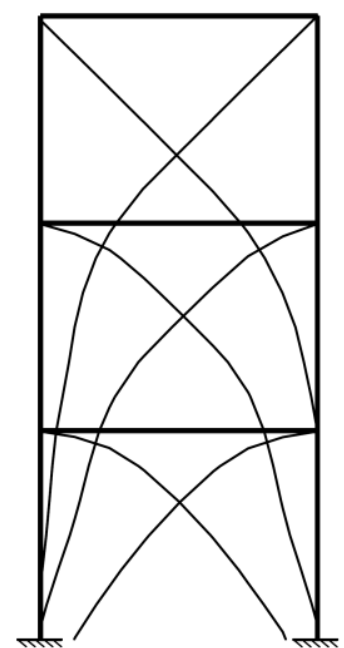

(b)

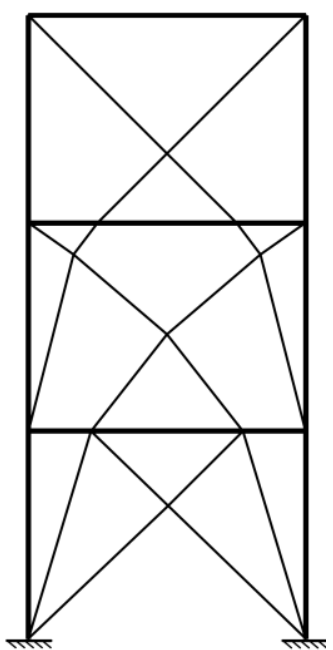

(c)

Fig. (5). Optimal results (a) Optimal truss-like continuum. (b) Optimal bracing systems (c) Modified bracing systems.

Table 4. Natural frequency $(\mathrm{rad} / \mathrm{s})$.

\begin{tabular}{|c|c|c|c|}
\hline Examples & 1 & 2 & 3 \\
\hline Braced Frame & 8.32 & 41.2 & 117 \\
\hline Braced Frame/Origin Frame & 1.87 & 2.20 & 2.90 \\
\hline
\end{tabular}

Fig. (6a). There are 14 groups of wide flange sections (American Institute of Steel Construction design code) used, which are shown in Table 3. Frame is divided by 114 beam elements; and truss-like continuum is meshed by $10 \times 18$ rectangular elements as shown in Fig. (6b). The optimal truss-like continuum distribution is shown in Fig. (7a), from which the optimal bracing system is established in Fig. (7b). Furthermore, this braced frame is converted to this one shown in Fig. (7c) as final optimal structure. 

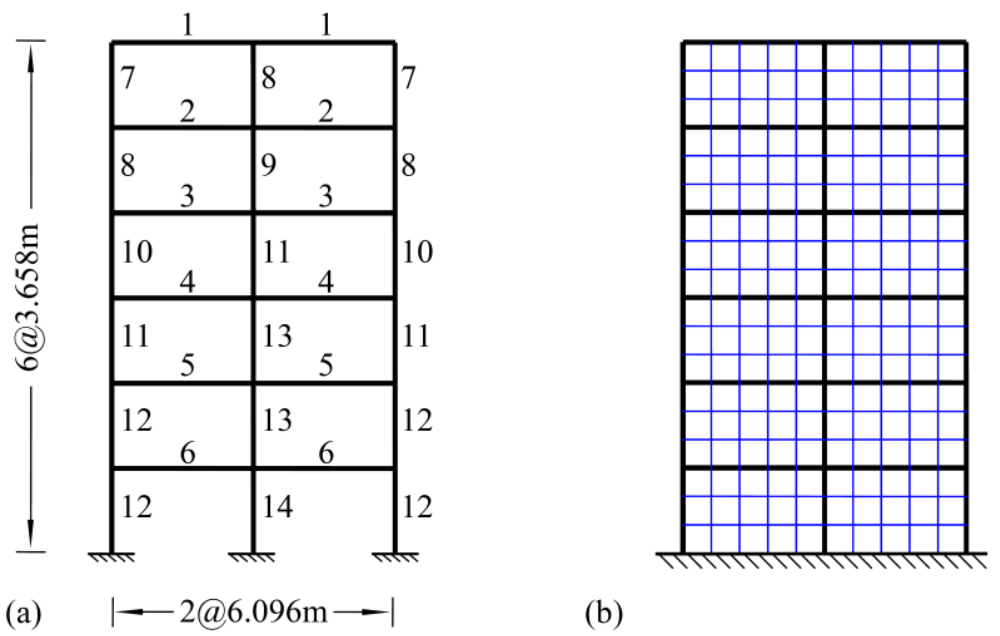

(b)

Fig. (6). 2-bay, 6-story frame (a) Structure and the number of member groups. (b) Calculation model.

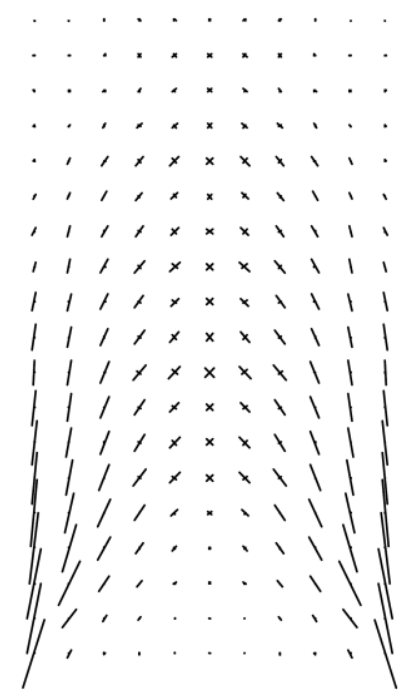

(a)

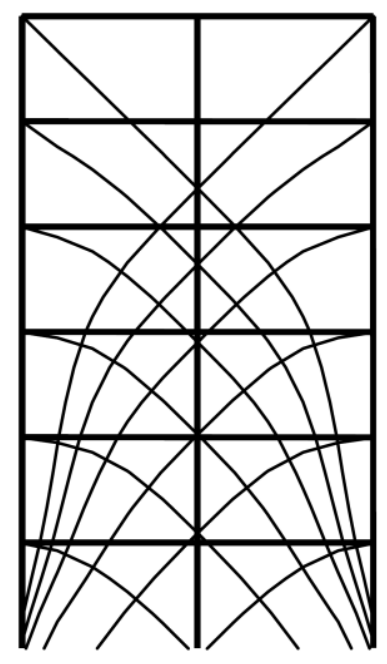

(b)

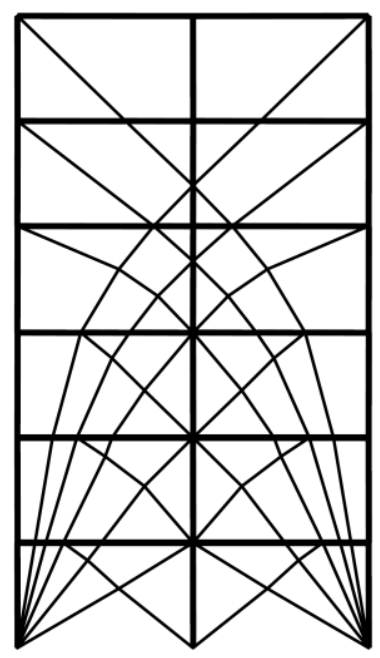

(c)

Fig. (7). Optimal results (a) Optimal truss-like continuum. (b) Optimal bracing systems (c) Modified bracing systems.

To verify the effect of optimization, the natural frequencies of frame with and without bracing systems are calculated. The material volumes of braced frame increase only $5 \%$ in the all examples. However the natural frequency of the braced frame is increased considerably as shown in Table 4.

\section{CONCLUSION}

An optimization method of frame bracing system to maximize structural natural frequency is presented. The topology of frame bracing system is optimized using trusslike material model. With 5\% material increase, the structural natural frequencies are increased greatly.

The optimization method based on truss-like continuum material model is highly effective.

\section{CONFLICT OF INTEREST}

The authors confirm that this article content has no conflict of interest.

\section{ACKNOWLEDGEMENTS}

This work is financially supported by the National Natural Science Foundation of China (No. 11172106).

\section{REFERENCE}

[1] R. Grandhi, "Structural optimization with frequency constraints-a review", AIAAJ, vol. 31, pp. 2296-2303, Dec. 1993.

[2] A. R. Mijar, C. C. Swan, J. S. Arora, and I. Kosaka, "Continuum topology optimization for concept design of frame bracing systems", J. Struct. Eng., vol. 124, pp. 541-550, May 1998.

[3] Q. Qing, Y. M. Xie, and P. S. Grant, "Optimal topology design of bracing systems for multistory steel frames", J. Struct. Eng., vol. 126, pp. 823-829, July 2000.

[4] X. Pan, J. Hao, and J Gao. "Study of adding cover-plate used for the single diagonal eccentrically braced steel frames", Open Civil Eng. J., vol. 5, pp. 143-153, June 2011

[5] A. G. M. Michell, "The limits of economy of material in frame structure", Phil. Mag., vol. 8, pp. 589-597, July 1904.

[6] M. P. Bendsøe, and N. Kikuchi, "Generating optimal topologies in structural design using a homogenization method", Comp. Methods Appl. Mech. Eng., vol. 71, pp. 197-224, Nov. 1988.

[7] L. H. Tenek, and I. Hagiwara, "Static and vibrational shape and topology optimization using homogenization and mathematical programming", Comput. Methods Appl. Mech. Eng., vol. 109, pp. 143-154, Oct. 1993. 
[8] X. Huang, Z. H. Zuo, and Y. M. Xie, "Evolutionary topological optimization of vibrating continuum structures for natural frequencies", Comp. Struct., vol. 88, pp. 357-364, March 2010.

[9] Y. M. Xie, and G. P. Steven, "Evolutionary structure optimization for dynamic problems", Comp. Struct., vol. 58, pp. 1067-1073, March 1996.

[10] J. A. Sethian, and A. Wiegmann, "Structural boundary design via level set and immersed interface methods", J. Comp. Phys., vol. 163, pp. 489-528, Sept. 1999.

[11] M. Bruggi, G. Milani, and A. Taliercio, "Simple topology optimization strategy for the FRP reinforcement of masonry walls in two-way bending", Comp. Struct., vol. 138, pp. 86-101, July 2014.

[12] M. Bruggi, G. Milani, and A. Taliercio, "Design of the optimal fiber-reinforcement for masonry structures via topology optimization", Int. J. Solids Struct., vol. 50, pp. 2087-2106, June 2013.
[13] H. A. Eschenauer, and N. Olhoff, "Topology optimization of continuum structures: a review", Appl. Mech. Rev., vol. 54, pp. 331389, July 2001.

[14] O. Sigmund, "Numerical instabilities in topology optimization: A survey on procedures dealing with checkerboards, meshdependencies and local minima", Struct. Multidisc. Optim., vol. 16, pp. 68-75, Aug 1998.

[15] K. Zhou, and X. Li, "Topology optimization of structures under multiple load cases using fiber-reinforced composite material model", Comp. Mech., vol. 38, pp. 163-170, July 2006.

[16] K. Zhou, and X. Li, "Topology optimization for minimum compliance under multiple loads based on continuous distribution of members", Struct. Multidisc. Optim., vol. 37, pp. 49-56, Dec 2008.

[17] K. Svanberg, "The method of moving asymptotes - a new method for structural optimization", Int. J. Numer. Methods Eng., vol. 24, pp. 359-373, Feb. 1987.

(c) Zhou and Chen; Licensee Bentham Open.

This is an open access article licensed under the terms of the Creative Commons Attribution Non-Commercial License (http://creativecommons.org/licenses/ by-nc/3.0/) which permits unrestricted, non-commercial use, distribution and reproduction in any medium, provided the work is properly cited. 